MATHEMATICS OF COMPUTATION

Volume 73, Number 246, Pages 595-611

S 0025-5718(03)01601-6

Article electronically published on October 27, 2003

\title{
FINITE DIFFERENCE METHOD FOR A COMBUSTION MODEL
}

\author{
LUNG-AN YING
}

\begin{abstract}
We study a projection and upwind finite difference scheme for a combustion model problem. Convergence to weak solutions is proved under the Courant-Friedrichs-Lewy condition. More assumptions are given on the ignition temperature; then convergence to strong detonation wave solutions or to weak detonation wave solutions is proved.
\end{abstract}

\section{INTRODUCTION}

Chemical reaction processes in fluids are determined by the conservation laws and the internal mechanism in reaction zones. Detonation waves travel at a high speed, and the scale of reaction zones may be orders of magnitude smaller than the fluid dynamical space scales. As a result the governing systems of equations are stiff. It was observed that even stable scheme and a reasonable grid may cause spurious numerical results. Sometimes the wave travelled one grid point per time step, which was qualitatively incorrect [5], [10], 11], [13].

One natural approach to deal with the problems in numerical computation is applying very fine meshes to simulate the internal mechanism in reaction zones. However, another strategy has been a subject of study by many authors, [1], [2], [8]: that is, to develop a method on a grid in which the resolution is not enough with respect to the short reacting time, but is fine enough with respect to the fluid dynamics. One cannot expect to predict the effects associated with the detailed structure of detonation fronts, but the detonation waves can be captured with correct speeds by using these methods. The aim of this paper is to study some convergence problems for this kind of methods.

We will study the problem for Majda's simplified combustion model [12], which is a $2 \times 2$ system, where a "lumped variable" are introduced to represent density, velocity and temperature. In addition we will consider the Chapman-Jouguet model for this $2 \times 2$ system; that is, the rate of chemical reaction is assumed to be infinity, and the effects of viscosity are neglected. In this model the width of reaction zones is zero and frame fronts are sharp. We think it is a typical model, which reflects some basic properties of the complete system, to study convergence problems of difference schemes for combustion problems.

Together with an upwind scheme we will consider the projection method, which has been applied by some authors. It is a fractional step method. In this method the numerical solution at each time level is computed in two steps. In the first step the upwind scheme is applied to a homogeneous conservation law, which causes

Received by the editor November 12, 2001 and, in revised form, October 22, 2002.

2000 Mathematics Subject Classification. Primary 65M06, 35L65, 76M20, 80A25.

Key words and phrases. Combustion, finite difference method, detonation wave, stiff equation. 
chemically nonequilibrium data. In the second step the nonlinear ODE describing the reacting progress is solved. If the numerical time step is much greater than the reacting time, the ODE solver essentially reduces to a projection operator, which coincides with the Chapman-Jouguet model, where the rate of chemical reaction is infinity.

The main difficulty lies in stiffness of the system. Numerical diffusion and smearing is introduced in the first step. Based on this smeared result, the result of the second step is sensitive to the choice of ignition temperature. On the other hand ignition temperature might be applied as a control parameter to the numerical results. This problem has been studied in 2. It was proved that if the approximate solutions tended to piecewise constant weak detonation waves, then the ignition temperature had to be less than a number $u_{l *}-q$, which will be explained later on in this paper. Therefore a necessary condition for generating weak detonation waves was proved. As a result, if one hopes to get a piecewise constant strong detonation wave, the assumed ignition temperature should be no less than it.

To overcome the difficulty of choosing the assumed ignition temperature, a random projection method was developed by Bao and Jin [1. The ignition temperature was assumed to be a random number with uniform distribution in the projection scheme. Strong detonation waves were obtained in one and two dimensional flows with chemical reaction. Asymptotic stability of this scheme was proved for a scalar equation with a stiff source term. Therefore the approach in [1 is effective for computing strong detonation waves. Meanwhile some problems have arisen. Why does a random number with uniform distribution yield strong detonation waves? What would happen if the distribution is not uniform? As a particular case, what are the results for different deterministic ignition temperatures?

We will answer part of these questions in this paper. We will give some sufficient conditions and prove convergence rigorously. It will be proved that under some assumptions the sequence of approximate solutions tends to a weak solution. Moreover, it will be proved that if the ignition temperature is no less than $u_{l *}-q$, then the limit is a piecewise constant strong detonation wave solution. Therefore this condition is not only necessary but also sufficient. We will also give a sufficient condition for generating weak detonation waves, and prove it.

We remark that although the results in this paper, like the usual convergence investigation, are on the limits of approximate solutions as the mesh sizes go to zero, it does not mean we study high resolution schemes here. We have assumed that the reacting time is zero, so the system is stiff for any positive mesh sizes, and the results are on the behavior of numerical solutions to a system with a stiff source term.

Let us state our problem. We consider the following Majda model for combustion 12]:

$$
\begin{gathered}
\frac{\partial(u+q z)}{\partial t}+\frac{\partial f(u)}{\partial x}=0 \\
\frac{\partial z}{\partial t}=-K \phi(u) z
\end{gathered}
$$

where $u$ is a "lumped variable", representing density, velocity and temperature, $z \in[0,1]$, representing the fraction of unburnt gas, $q>0$ is a constant, representing the binding energy, $K>0$ is a constant, representing the rate of chemical reaction, 


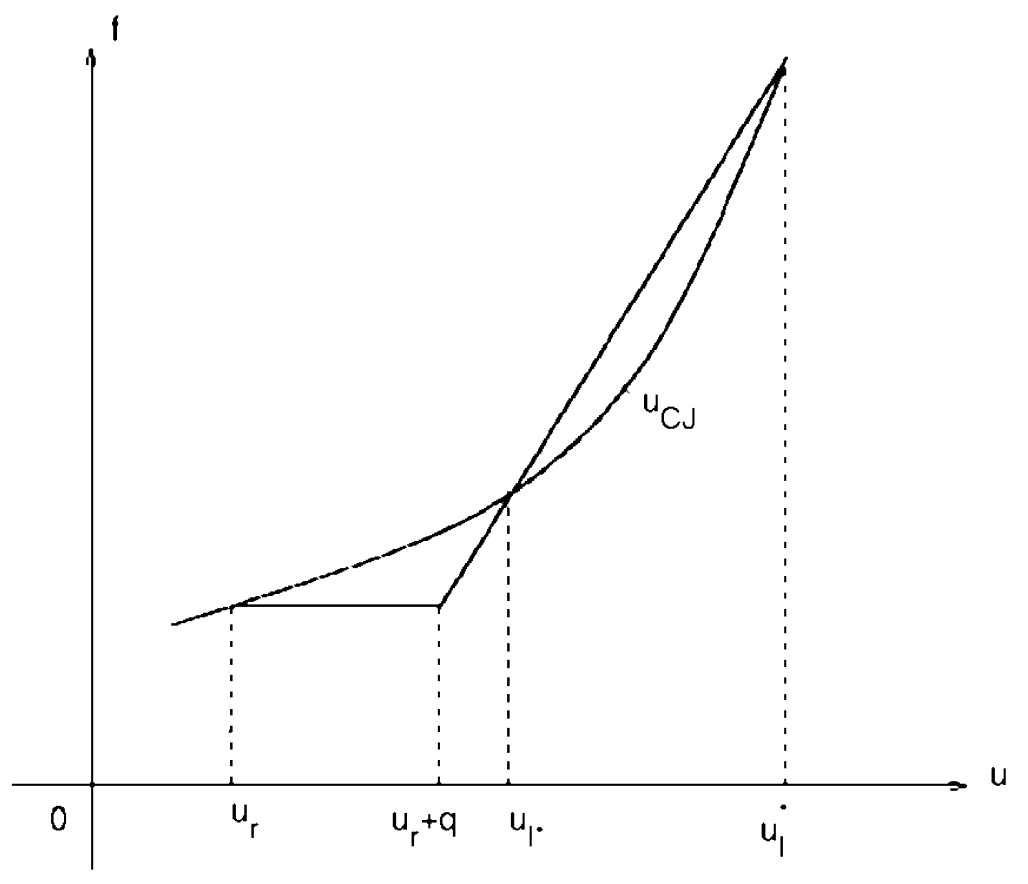

FIGURE 1.

$f^{\prime}>0, f^{\prime \prime} \geq a_{0}>0$

$$
\phi(u)= \begin{cases}1, & u>U_{i}, \\ 0, & u<U_{i},\end{cases}
$$

and $U_{i}$ is a constant, representing the ignition temperature.

We assume the initial condition

$$
\left.u\right|_{t=0}=u_{0}(x),\left.\quad z\right|_{t=0}=z_{0}(x),
$$

where naturally we assume that $z_{0} \in[0,1]$ and $z_{0}(x)=0$ for a certain $x$ if $u_{0}(x)>$ $U_{i}$.

Let $K \rightarrow+\infty$ formally; then we get $\frac{\partial z}{\partial t} \leq 0, \phi(u) z=0$, and if $u<U_{i}$ then $\frac{\partial z}{\partial t}=0$. Therefore (2) is replaced by

$$
z(x, t)= \begin{cases}0, & \sup _{0 \leq \tau \leq t} u(x, \tau)>U_{i}, \\ z_{0}(x), & \sup _{0 \leq \tau \leq t} u(x, \tau)<U_{i}\end{cases}
$$

and

$$
\frac{\partial z}{\partial t} \leq 0
$$

A global existence theorem for the problem (1), (4), (5), (3) was proved in [14].

One special case for the above problem is the Riemann problem, where the initial condition is

$$
\left.u\right|_{t=0}=\left\{\left.\begin{array}{ll}
u_{l}, & x<0, \\
u_{r}, & x>0,
\end{array} \quad z\right|_{t=0}= \begin{cases}0, & x<0, \\
1, & x>0,\end{cases}\right.
$$

where $u_{r}<U_{i} \leq u_{l}$ and $u_{l}>u_{r}+q$. 
The travelling wave solution for the Riemann problem is the following:

$$
u(x, t)=\left\{\begin{array}{ll}
u_{l}, \quad x<s t, \\
u_{r}, \quad x>s t,
\end{array} \quad z(x, t)= \begin{cases}0, & x<s t, \\
1, & x>s t,\end{cases}\right.
$$

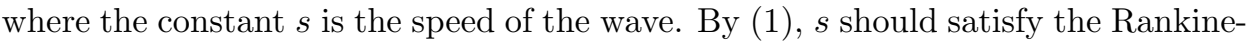
Hugoniot condition,

$$
s=\frac{f\left(u_{l}\right)-f\left(u_{r}\right)}{u_{l}-\left(u_{r}+q\right)} .
$$

By the assumption of $f$ there exists $u_{C J}>u_{r}+q$ such that

$$
f^{\prime}\left(u_{C J}\right)=\frac{f\left(u_{C J}\right)-f\left(u_{r}\right)}{u_{C J}-\left(u_{r}+q\right)} .
$$

$f^{\prime}\left(u_{C J}\right)$ is the minimum value of $s$, and the corresponding travelling wave for $u_{l}=$ $u_{C J}$, is called a "CJ detonation wave". For a given $s \in\left(f^{\prime}\left(u_{C J}\right), \infty\right)$ there are two values of $u_{l}$ corresponding to $s$. Let them be $u_{l}^{*}>u_{C J}$ and $u_{l *}<u_{C J}$, and the corresponding travelling waves be called "strong detonation waves" and "weak detonation waves" respectively (Figure 1). We will always assume that $u_{l}=u_{l}^{*}$ in (6) later on. (7) is a solution of the Riemann problem, but the solutions are not unique. For example

$$
u(x, t)=\left\{\begin{array}{ll}
u_{l}, & x<s_{1} t, \\
u_{m}, & s_{1} t<x<s_{2} t \\
u_{r}, & x>s_{2} t,
\end{array} \quad z(x, t)= \begin{cases}0, & x<s_{2} t, \\
1, & x>s_{2} t,\end{cases}\right.
$$

where $u_{l *}>u_{m}>u_{r}+q, u_{m}>U_{i}$, and

$$
s_{1}=\frac{f\left(u_{l}\right)-f\left(u_{m}\right)}{u_{l}-u_{m}}, \quad s_{2}=\frac{f\left(u_{m}\right)-f\left(u_{r}\right)}{u_{m}-\left(u_{r}+q\right)} .
$$

It is a solution with two waves, where a weak detonation wave is followed by a shock wave. The value of $u_{m}$ is also not unique.

Since the solutions are not unique, different parameters in the numerical scheme lead to different solutions. We are going to give some sufficient conditions and prove that the approximate solutions of the difference scheme converge to some different limits. We state our main results and assumptions as the follows.

We study a projection and upwind finite difference scheme, which will be given in the next section. Let $\Delta x, \Delta t$ be the step sizes. The numerical ignition temperature at time $n \Delta t$ is denoted by $U_{i}^{n}$. Then we consider a series of approximate solutions to the Riemann problem and assume that $\Delta x, \Delta t \rightarrow 0$. If $U_{i}^{n}$ is taken as a random number, then we require that $U_{i}^{n} \in(U-q, U)$, where $U \in\left[u_{r}+q, u_{l}\right]$. If $U_{i}^{n}$ is fixed, which can be regarded as a special case of a random number, we denote $U_{i}=U=U_{i}^{n} \in\left(u_{r}, u_{l}\right)$.

Theorem 1.1. If

$$
\frac{\Delta t}{\Delta x} \max _{u \in\left[u_{r}, u_{l}+2 q\right]} f^{\prime}(u) \leq \frac{q}{u_{l}+2 q-u_{r}},
$$

then there is a subsequence of the approximate solutions converging pointwise almost everywhere for $x \in(-\infty, \infty), t \in(0, \infty)$. If $(u, z)$ is the limit, then there is a curve $\Gamma: x=l(t)$ such that $u$ is an entropy solution of the scalar conservation law

$$
\frac{\partial u}{\partial t}+\frac{\partial f(u)}{\partial x}=0
$$


for $x \neq l(t), z=1$ for $x>l(t)$, and $z=0$ for $x<l(t)$. Moreover $(u, z)$ is the weak solution to (1),

$$
z(x, t)= \begin{cases}0, & \sup _{0 \leq \tau \leq t} u(x, \tau)>U, \\ z_{0}(x), & \sup _{0 \leq \tau \leq t} u(x, \tau)<U,\end{cases}
$$

(5) and (6) .

Theorem 1.2. Under the assumption of Theorem 1.1 if $U_{i}$ is fixed, assume that $U_{i} \in\left[u_{l_{*}}-q, u_{l}^{*}\right)$; if $U_{i}^{n}$ is random, assume that $U \in\left[u_{l_{*}}, u_{l}^{*}\right)$, Then as $\Delta x, \Delta t \rightarrow 0$, the approximate solution converges to a unique strong detonation limit (7). For the random case the conclusions should be understood in the sense of probability 1.

Theorem 1.3. If $U_{i}$ is fixed and

$$
\frac{\Delta t}{\Delta x} \max \left(f^{\prime}\left(u_{l}^{*}\right), \frac{f\left(u_{l}^{*}\right)-f\left(u_{l *}-q\right)}{u_{l}^{*}-u_{l *}}\right) \leq 1,
$$

then there is a constant $u_{w} \in\left(u_{r}, u_{l *}-q\right)$ such that if $U_{i} \in\left(u_{r}, u_{w}\right]$, there is a subsequence of the approximate solutions converging pointwise almost everywhere for $x \in(-\infty, \infty), t \in(0, \infty)$. Let $(u, z)$ be the limit; then there is a curve $\Gamma: x=$ $l(t)$ such that $u$ is an entropy solution of the scalar conservation law

$$
\frac{\partial u}{\partial t}+\frac{\partial f(u)}{\partial x}=0
$$

for $x \neq l(t), z=1$ for $x>l(t)$, and $z=0$ for $x<l(t)$. Moreover $(u, z)$ is a weak solution to (11), (10), (5), (6), and the discontinuity $\Gamma$ is a weak detonation wave in the following sense: (a) The slope of characteristics for $x>l(t)$ is less than the slope of this discontinuity, and the downward characteristics for $x<l(t)$ neighboring this discontinuity intersect it. (b) The Rankine-Hugoniot condition for weak detonation waves holds on $\Gamma$.

We will prove the above theorems in the following sections. For Theorem 1.3 we will give the exact value of $u_{w}$ and state the Rankine-Hugoniot condition precisely. Finally, we will compare our results with the results in [12], which is about continuous travelling detonation waves.

\section{DifFERENCE SCHEME AND CONVERGENCE TO WEAK SOLUTIONS}

We consider a fractional step method for the system of equations (11), (4), (5) with initial conditions (3) or (6), where the convection and the chemical reaction are split by a three-step procedure, and the solver of the convection part is an upwind scheme.

Step 1.

$$
\frac{\tilde{u}_{j}^{n}-u_{j}^{n}}{\Delta t}+\frac{f\left(u_{j}^{n}\right)-f\left(u_{j-1}^{n}\right)}{\Delta x}=0
$$

Step 2.

$$
z_{j}^{n+1}= \begin{cases}0, & \tilde{u}_{j}^{n} \geq U_{i}^{n} \\ z_{j}^{n}, & \tilde{u}_{j}^{n}<U_{i}^{n}\end{cases}
$$

Step 3.

$$
u_{j}^{n+1}=\tilde{u}_{j}^{n}-q\left(z_{j}^{n+1}-z_{j}^{n}\right),
$$


where $u_{j}^{n}=u(j \Delta x, n \Delta t), z_{j}^{n}=z(j \Delta x, n \Delta t)$, and $\tilde{u}_{j}^{n}$ is an intermediate variable. The scheme is conservative:

$$
\frac{u_{j}^{n+1}-u_{j}^{n}}{\Delta t}+q \frac{z_{j}^{n+1}-z_{j}^{n}}{\Delta t}+\frac{f\left(u_{j}^{n}\right)-f\left(u_{j-1}^{n}\right)}{\Delta x}=0 .
$$

We will prove some estimates for the scheme applied to the Riemann problem, and study the limit of approximate solutions as $\Delta t \rightarrow 0$ and $\Delta x \rightarrow 0$.

Lemma 2.1. If (9) holds, then

$$
z_{j}^{n} \in[0,1], \quad u_{j}^{n} \in\left[u_{r}, u_{l}+2 q\right], \quad \forall j, n .
$$

Proof. Obviously $z_{j}^{n} \in[0,1]$, and $u_{j}^{n} \geq u_{r}$. 16 is true for $n=0$. Let us assume that it is true for a certain $n \geq 0$. We notice that $f^{\prime}>0$, and if $\frac{\Delta t}{\Delta x} \max f^{\prime} \leq 1$, the scheme (12) is monotone. The condition (91) is stronger. We make use of the properties of monotone schemes (see [4]) to obtain $\tilde{u}_{j}^{n} \leq \max _{k} u_{k}^{n} \leq u_{l}+2 q$. For a given $j$, if $u_{j}^{n+1}=\tilde{u}_{j}^{n}$, then (16) is verified for $u^{n+1}$. If $u_{j}^{n+1}>\tilde{u}_{j}^{n}$, then $z_{j}^{n+1}=0$ and $z_{j}^{n}=1$. Consequently $z_{j}^{n-1}=1$ if $n \geq 1$. Then, by (13), (14), $u_{j}^{n}=\tilde{u}_{j}^{n-1}<U_{i}^{n-1}<U$. If $n=0$, then $u_{j}^{n}=u_{r}<U$, too. Therefore by (12)

$$
\tilde{u}_{j}^{n}<U+\frac{\Delta t}{\Delta x} \max _{u \in\left[u_{r}, u_{l}+2 q\right]} f^{\prime}(u) \cdot\left(u_{l}+2 q-u_{r}\right) .
$$

The condition (9) implies

$$
u_{j}^{n+1}<U+2 q \leq u_{l}+2 q,
$$

and so (16) is verified for $n+1$.

Lemma 2.2. Under the condition (9) there is an integer $j_{0}^{n}$ for each $n$ such that $z_{j}^{n}=0, u_{j}^{n} \geq U$ for $j \leq j_{0}^{n}$, and $z_{j}^{n}=1, u_{j}^{n} \leq u_{j-1}^{n}, u_{j}^{n} \leq U$ for $j>j_{0}^{n}$.

Proof. It is true for $n=0$. We assume that it is true for a certain $n \geq 0$. Because the scheme is monotone, $\tilde{u}_{j}^{n} \geq U$ for $j \leq j_{0}^{n}$; then $u_{j}^{n+1}=\tilde{u}_{j}^{n} \geq U$ and $z_{j}^{n+1}=0$. Let $j_{0}^{n+1}$ be an index such that $\tilde{u}_{j}^{n} \geq U_{i}^{n}$ for all $j \leq j_{0}^{n+1}$ and $\tilde{u}_{j}^{n}<U_{i}^{n}$ for $j=j_{0}^{n+1}+1$. Then $j_{0}^{n+1} \geq j_{0}^{n}$. For $j=j_{0}^{n}+1, \cdots, j_{0}^{n+1}$, we have $z_{j}^{n}=1$ and $z_{j}^{n+1}=0$; hence $u_{j}^{n+1}=\tilde{u}_{j}^{n}+q \geq U$. Again because the scheme is monotone, $\tilde{u}_{j}^{n} \leq \tilde{u}_{j-1}^{n}$ for $j \geq j_{0}^{n+1}+2$. Since $\tilde{u}_{j}^{n}<U_{i}^{n}$ for $j=j_{0}^{n+1}+1$, all $\tilde{u}_{j}^{n}<U_{i}^{n}$ for $j \geq j_{0}^{n+1}+1$. Thus $z_{j}^{n+1}=z_{j}^{n}=1$ and $u_{j}^{n+1}=\tilde{u}_{j}^{n}<U_{j}^{n}<U$. Finally we have $u_{j}^{n+1} \geq U \geq u_{j+1}^{n+1}$ with $j=j_{0}^{n+1}$, so $u_{j}^{n+1}$ is monotone decreasing for all $j \geq j_{0}^{n+1}$.

Lemma 2.3. $u_{j}^{n+1} \geq u_{j}^{n}$ for $j>j_{0}^{n}$.

Proof. Since $f^{\prime}>0$ and $u_{j}^{n} \leq u_{j-1}^{n}$, equation (12) implies $\tilde{u}_{j}^{n} \geq u_{j}^{n}$. Then by (14) we have $u_{j}^{n+1} \geq \tilde{u}_{j}^{n}$.

If $U_{i}<u_{l *}-q$ and is fixed, then we can improve our maximum norm estimate in Lemma 2.1 and the CFL condition (9).

Lemma 2.4. If $U_{i}<u_{l *}-q$ and (11) holds, then $U_{i}+q \leq u_{j}^{n} \leq u_{l}^{*}$ for $j \leq j_{0}^{n}$, and $u_{j}^{n}<U_{i}$ for $j>j_{0}^{n}$. 
Proof. For simplicity we denote $j_{0}=j_{0}^{n}$. We argue by induction. We assume that the conclusion is true for a certain $n$. The function $\frac{f\left(u_{l}^{*}\right)-f(u)}{u_{l}^{*}-u-q}$ is monotonically increasing with respect to $u$ for $u<u_{l}^{*}-q$; thus, by the CFL condition (11),

$$
\begin{aligned}
\tilde{u}_{j_{0}+1}^{n} & \leq u_{j_{0}+1}^{n}+\frac{u_{l}^{*}-U_{i}-q}{f\left(u_{l}^{*}\right)-f\left(U_{i}\right)}\left(f\left(u_{j_{0}}^{n}\right)-f\left(u_{j_{0}+1}^{n}\right)\right) \\
& \leq u_{j_{0}+1}^{n}+\frac{u_{l}^{*}-U_{i}-q}{f\left(u_{l}^{*}\right)-f\left(U_{i}\right)}\left(f\left(u_{l}^{*}\right)-f\left(u_{j_{0}+1}^{n}\right)\right) \equiv \Phi\left(u_{j_{0}+1}^{n}\right),
\end{aligned}
$$

where we notice that $U_{i}<u_{l *}-q<u_{l}^{*}-q$. Then for $\xi \in\left[u_{r}, U_{i}\right]$,

$$
\Phi^{\prime}(\xi)=1-\frac{u_{l}^{*}-U_{i}-q}{f\left(u_{l}^{*}\right)-f\left(U_{i}\right)} f^{\prime}(\xi) \geq 1-\frac{u_{l}^{*}-U_{i}-q}{f\left(u_{l}^{*}\right)-f\left(U_{i}\right)} f^{\prime}\left(U_{i}\right) \geq 0 .
$$

By the induction assumption we have $u_{j_{0}+1}^{n}<U_{i}$; therefore

$$
\tilde{u}_{j_{0}+1}^{n} \leq \Phi\left(u_{j_{0}+1}^{n}\right) \leq \Phi\left(U_{i}\right) \leq u_{l}^{*}-q .
$$

The scheme (12) is monotone, so $U_{i}+q \leq u_{j}^{n+1}=\tilde{u}_{j}^{n} \leq u_{l}^{*}$ and $z_{j}^{n+1}=0$ for $j \leq j_{0}^{n}$. Let $j_{0}^{n+1} \geq j_{0}^{n}$ be an index such that $\tilde{u}_{j}^{n} \geq U_{i}$ for $j_{0}^{n}<j \leq j_{0}^{n+1}$ and $\tilde{u}_{j}^{n}<U_{i}$ for $j=j_{0}^{n+1}+1$; then $u_{j}^{n+1}=\tilde{u}_{j}^{n}+q \in\left[U_{i}+q, u_{l}^{*}\right]$ and $z_{j}^{n+1}=0$ for $j_{0}^{n}<j \leq j_{0}^{n+1}$. If $j>j_{0}^{n+1}$, then $\tilde{u}_{j}^{n}<U_{i}$, so $u_{j}^{n+1}=\tilde{u}_{j}^{n}<U_{i}$. Thus the induction is complete.

We have $0=j_{0}^{0} \leq j_{0}^{1} \leq \cdots \leq j_{0}^{n} \leq j_{0}^{n+1} \leq \cdots$. Define a subsequence $j_{0}^{0}, j_{0}^{n_{1}}, j_{0}^{n_{2}}, \cdots$ such that $j_{0}^{0}=j_{0}^{1}=\cdots=j_{0}^{n_{1}-1}$ and $j_{0}^{n_{1}}>j_{0}^{n_{1}-1}$, and so on. We connect points $\left(j_{0}^{n_{k}} \Delta x, n_{k} \Delta t\right),\left(j_{0}^{n_{k+1}} \Delta x, n_{k+1} \Delta t\right)$ by line segments, $k=0,1, \cdots$, then get a curve, denoted by $x=l_{\Delta x}(t)$.

Lemma 2.5. $l_{\Delta x}^{\prime}(t)>0$, and $l_{\Delta x}(t)$ is bounded for all $\Delta x$ on $t \in[0, T]$, where $T$ is an arbitrary positive number.

Proof. For each $n$, if $j>n$; then $u_{j}^{n} \equiv u_{r}$ and $z_{j}^{n} \equiv 1$, so $j_{0}^{n} \leq n$. By the CFL condition, $l_{\Delta x}(t)$ is bounded.

Let $\Delta x, \Delta t \rightarrow 0$, then there is a subsequence of $l_{\Delta x}(t)$ converging pointwise to a curve $\Gamma: x=l(t)$ with $l^{\prime}(t) \geq 0$ and $l \in B V(0, T)$. However, we hope to get uniform convergence. To this end we define new coordinates

$$
\left(\begin{array}{c}
x^{\prime} \\
t^{\prime}
\end{array}\right)=\left(\begin{array}{cc}
\cos \frac{\pi}{4} & \sin \frac{\pi}{4} \\
-\sin \frac{\pi}{4} & \cos \frac{\pi}{4}
\end{array}\right)\left(\begin{array}{c}
x \\
t
\end{array}\right) .
$$

Let the curve be $t^{\prime}=\tilde{l}_{\Delta x}\left(x^{\prime}\right)$ in the new coordinates. Since $0<l_{\Delta x}^{\prime}<+\infty$, we have $\left|\frac{d}{d x^{\prime}} \tilde{l}_{\Delta x}\left(x^{\prime}\right)\right| \leq 1$, which gives uniform convergence. The limit is denoted by $\tilde{l}\left(x^{\prime}\right)$. Let $\varepsilon>0$; then $\tilde{l}\left(x^{\prime}\right) \pm \varepsilon$ defines an $\varepsilon$-neighborhood of this curve, denoted by $N_{\varepsilon}$. Let $\Omega_{+}=\{(x, t) ; x>l(t)\}$ and $\Omega_{-}=\{(x, t) ; x<l(t)\}$.

We extend $u_{j}^{n}$ and $z_{j}^{n}$ by constants on $(j \Delta x,(j+1) \Delta x] \times(n \Delta t,(n+1) \Delta t]$, denoted by $u_{\Delta x}$ and $z_{\Delta x}$. On $\Omega_{+} \backslash N_{\varepsilon}, u_{\Delta x}$ is bounded in $B V$. There is a subsequence converging in $L^{1}$, and the limit $u$ is in $B V . u$ is monotone. On $\Omega_{-} \backslash N_{\varepsilon}, u_{\Delta x}$ is bounded in $L^{\infty}$. There is a subsequence of the above subsequence weakly converging in $L^{p}, p>1$. Letting $\varepsilon \rightarrow 0$, we get a sequence of $u_{\Delta x}$ converging for all $\varepsilon$. Let $u$ be the limit. There is a family of Young measures $\left\{\nu_{x, t}\right\}$ associated with the sequence (see [9], for example). To see the strong convergence in $\Omega_{-} \backslash N_{\varepsilon}$, it suffices 
to prove the Young measures are Dirac $\delta$ functions. Consider an arbitrary $t_{0}>0$. The theory of compensated compactness is applied to the difference scheme

$$
\frac{v_{j}^{n+1}-v_{j}^{n}}{\Delta t}+\frac{f\left(v_{j}^{n}\right)-f\left(v_{j-1}^{n}\right)}{\Delta x}=0,
$$

with initial data

$$
\begin{array}{cc}
v_{j}^{n_{0}}=u_{j}^{n_{0}} \quad \text { for } j \Delta x \leq l\left(t_{0}\right)-\varepsilon, \\
v_{j}^{n_{0}}=u_{r} \quad \text { for } j \Delta x>l\left(t_{0}\right)-\varepsilon .
\end{array}
$$

where $n_{0}=\left[\frac{t_{0}}{\Delta t}\right]$ and $[\cdot]$ is the integer part of a number. This is the upwind finite difference scheme for the conservation law

$$
\frac{\partial u}{\partial t}+\frac{\partial f(u)}{\partial x}=0
$$

It is easy to see that $v_{j}^{n}=u_{j}^{n}$ for all $j \leq\left(l\left(t_{0}\right)-\varepsilon\right) / \Delta x$ and $n \geq n_{0}$, and $v_{j}^{n}$ also converges weakly in $L^{p}$. Following the argument in [7], we can prove that the Young measures associated with $v_{j}^{n}$ are Dirac $\delta$ functions for all $x$ and $t$, and so are the Young measures associated with $u_{j}^{n}$ for $j \leq\left(l\left(t_{0}\right)-\varepsilon\right) / \Delta x$ and $n \geq n_{0}$. Since $l$ is monotone and $t_{0}$ is arbitrary, it is true for all $(x, t) \in \Omega_{-} \backslash N_{\varepsilon}$, and $\varepsilon$ is arbitrary.

Lemma 2.6. If there is a sequence of time steps with $\Delta x, \Delta t \rightarrow 0$, then there is a subsequence such that $u_{\Delta x}$ converges pointwise almost everywhere to $u$ on $t \in(0, T)$. $u$ is an entropy solution for $x<l(t)$ and $x>l(t)$, and

$$
\begin{array}{ll}
u \geq U, z=0, & \text { for }(x, t) \in \Omega_{-}, \\
u \leq U, z=1, \quad & \text { for }(x, t) \in \Omega_{+} .
\end{array}
$$

Proof. The conclusion follows from the above argument and Lemma 2.2

We are now ready to prove Theorem 1.1

Proof of Theorem 1.1. Let $\Omega=\{(x, t) ;-\infty<x<\infty, 0<t<\infty\}$ and $\varphi \in C_{0}^{\infty}(\Omega)$. By (15) we have

$$
\sum_{j=-\infty}^{\infty} \sum_{n=0}^{\infty}\left\{\left(u_{j}^{n}+q z_{j}^{n}\right) \frac{\varphi_{j}^{n+1}-\varphi_{j}^{n}}{\Delta t}+f\left(u_{j}^{n}\right) \frac{\varphi_{j+1}^{n}-\varphi_{j}^{n}}{\Delta x}\right\} \Delta x \Delta t=0 .
$$

Let $\Delta x, \Delta t \rightarrow 0$ for a given $\varepsilon>0$; then we get the limit

$$
\iint_{\Omega \backslash N_{\varepsilon}}\left\{(u+q z) \frac{\partial \varphi}{\partial t}+f(u) \frac{\partial \varphi}{\partial x}\right\} d x d t=O(\varepsilon)\|\varphi\|_{C_{0}^{1}} .
$$

Here we have used the maximum norm estimate in Lemma 2.1 and the fact that $\operatorname{meas}\left(\operatorname{supp} \varphi \bigcap N_{\varepsilon}\right)=O(\varepsilon) . \varepsilon$ is arbitrary, so

$$
\iint_{\Omega}\left\{(u+q z) \frac{\partial \varphi}{\partial t}+f(u) \frac{\partial \varphi}{\partial x}\right\} d x d t=0,
$$

which proves $(u, z)$ is a weak solution to (11). The other conclusions are obvious. Thus the proof is completed.

We have noticed that the solutions of this problem are not unique. 


\section{Convergence to Strong Detonation WAVES}

We will make more assumption in addition to the CFL condition (9); then we will prove the weak solution obtained in the previous section is a piecewise constant solution with a strong detonation wave. Let all assumptions of Theorem 1.2 be satisfied in this section. To prove the theorem we need the following lemmas.

Lemma 3.1. $u \equiv u_{r}$ on $\Omega_{+}$.

Proof. First of all, let us prove that

$$
u_{\min }^{-}-u_{\max }^{+} \geq q
$$

where $u_{\min }^{-}=\min _{(x, t) \in \Omega_{-}} u(x, t)$ and $u_{\max }^{+}=\max _{(x, t) \in \Omega_{+}} u(x, t)$. If $U_{i}$ is fixed, then $u \leq U_{i}$ on $\Omega_{+}$and $u \geq U_{i}+q$ on $\Omega_{-}$, so (17) holds. If $U_{i}^{n}$ is random, we set $u_{\max }^{+}=\inf \left\{v \in(U-q, U) ; P\left(U_{i}^{n}<v\right)>0\right\}$. We construct a $2 \varepsilon$-neighborhood $N_{2 \varepsilon}$. If $(x, t) \notin N_{2 \varepsilon}$, we take $t_{1}<t$ such that

$$
t-t_{1} \leq \frac{\varepsilon}{\max f^{\prime}}
$$

For a given $\delta>0$ when $\Delta x, \Delta t$ are sufficiently small, there is an $n_{0} \Delta t \in\left(t_{1}, t\right)$ such that $U_{i}^{n_{0}} \leq u_{\max }^{+}+\delta$; then $u_{j}^{n_{0}} \leq u_{\max }^{+}+\delta$ for all $j$ on $\Omega_{+} \backslash N_{\varepsilon}$. The scheme is monotone; therefore $u_{j}^{n} \leq u_{\max }^{+}+\delta$ holds for $n \geq n_{0}$ on $\Omega_{+} \backslash N_{\varepsilon}$. Since $u_{\Delta x}(x, t) \leq$ $u_{\max }^{+}+\delta$ for $(x, t) \in \Omega_{+} \backslash N_{2 \varepsilon}$, we have $u(x, t) \leq u_{\max }^{+}+\delta$. But $\delta$ is arbitrary, and $\varepsilon$ is also arbitrary, so we have $u(x, t) \leq u_{\max }^{+}$on $\Omega_{+}$. On the other hand, $u(x, t) \geq u_{\max }^{+}+q$ on $\Omega_{-}$. Therefore (17) holds.

Let $\left(x_{0}, t_{0}\right) \in \Omega_{+}$. The downward characteristic line starting from $\left(x_{0}, t_{0}\right)$ is denoted by $C\left(x_{0}, t_{0}\right)$, which is a function $x=g\left(t ; x_{0}, t_{0}\right)$ (see [6]). If $g\left(t ; x_{0}, t_{0}\right)>$ $l(t), \forall t \in\left(0, t_{0}\right)$, then $u$ is a constant on $C\left(x_{0}, t_{0}\right)$ and $u\left(x_{0}, t_{0}\right)=u_{r}$. If there is $t_{1} \in\left(0, t_{0}\right)$ such that $g\left(t_{1} ; x_{0}, t_{0}\right)=l\left(t_{1}\right)$, and $g\left(t ; x_{0}, t_{0}\right)>l(t)$ for $t \in\left(t_{1}, t_{0}\right)$, we construct all $C(l(\tau), \tau), \tau \leq t_{1}$. Let $g(t)=\max \left\{l(t), g\left(t ; x_{0}, t_{0}\right), g(t ; l(\tau), \tau), \forall \tau \leq\right.$ $\left.t_{1}\right\}$; then $g$ is continuous (Figure 2$)$. Also, $g^{\prime}(t) \in\left[0, f^{\prime}(U)\right]$, so $g \in W^{1, \infty}$.

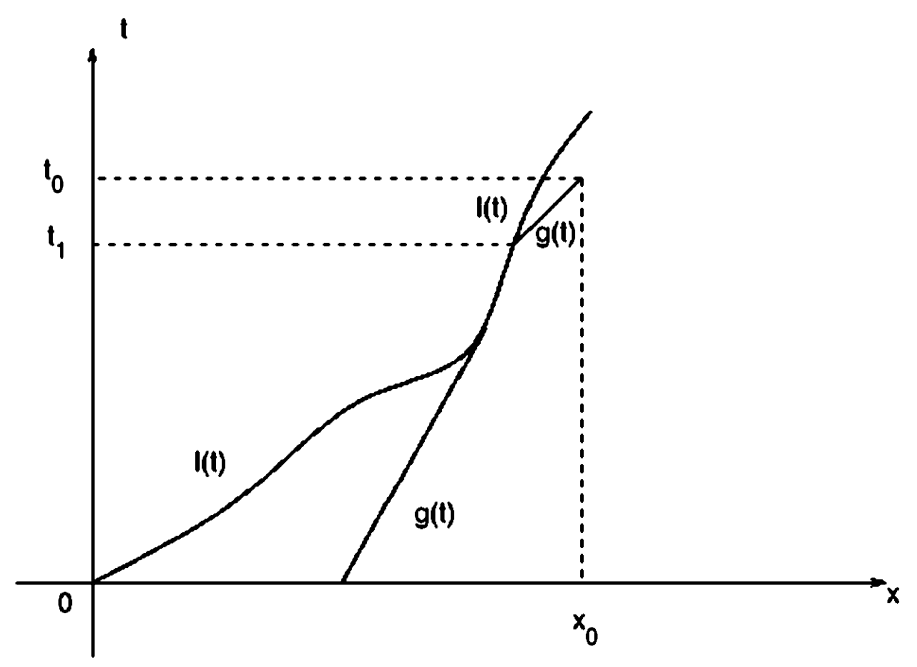

Figure 2. 
Let $v(t)=u(g(t)+0, t)$. Because $u \in B V$, the limit exists as $x \rightarrow g(t)+0$.

Let $S=\left\{t \in\left(0, t_{0}\right) ; g(t)=l(t)\right\}$. The derivatives of $l$ and $g$ exist almost everywhere. By the definition of $g$, the slope $f^{\prime}(v(t))$ of the characteristic line is greater than or equal to the slope of the curve $g(t)$. At a point $t \in S$, if both $l^{\prime}$ and $g^{\prime}$ exist, then $l^{\prime}(t)=g^{\prime}(t) \leq f^{\prime}\left(u_{\max }^{+}\right)$. On the other hand, if $x<l(t)$, then the slope of $C(x, t)$ is no less than $f^{\prime}\left(u_{\min }^{-}\right)$. Let $\delta>0$ be small enough so that $\frac{l(t)-l(\tau)}{t-\tau}<f^{\prime}\left(u_{\min }^{-}\right)$for $\tau \in[t-\delta, t]$; then $g(\tau ; x, t)<l(\tau)$ for all $\tau \in[t-\delta, t]$. As $x \rightarrow l(t)-0, g(\tau ; x, t)$ is increasing and the $\operatorname{limit}_{x \rightarrow l(t)-0} g(\tau ; x, t)$ exists. Now $\frac{x-g(t-\delta ; x, t)}{\delta}=f^{\prime}(u(x, t))$. Since $f^{\prime \prime}>0$, the limit of $u(x, t)$ also exists as $x \rightarrow l(t)-0$.

Since $u(g(t)+0), t)=u(g(t)-0, t)$ on characteristics, using the definition of weak solutions we get the Rankine-Hugoniot condition

$\int_{S} g^{\prime}(t)(u(g(t)+0, t)+q-u(g(t)-0, t)) d t=\int_{S}(f(u(g(t)+0, t))-f(u(g(t)-0, t))) d t$,

where the integral is zero for $t \notin S$. By the definition of $S$,

$$
0 \leq g^{\prime}(t) \leq f^{\prime}(u(g(t)+0, t)) .
$$

(17) implies that

$$
\begin{gathered}
f(u(g(t)+0, t))<f(u(g(t)-0, t)), \\
u(g(t)+0, t)+q \leq u(g(t)-0, t) .
\end{gathered}
$$

Consequently

$$
\begin{aligned}
& f(u(g(t)-0, t))-f(u(g(t)+0, t)) \\
> & f^{\prime}(u(g(t)+0, t))(u(g(t)-0, t)-u(g(t)+0, t)-q) \\
\geq & g^{\prime}(t)(u(g(t)-0, t)-u(g(t)+0, t)-q) .
\end{aligned}
$$

Therefore meas $S=0$.

We define a curve $\Gamma^{\varepsilon}: x=l(t)+\varepsilon$. We have $\Gamma^{\varepsilon} \subset \Omega_{+}$. From each point on it we can construct $C(x, t)$. Similar to $g(t)$ and $v(t)$ we have $g^{\varepsilon}(t)$ and $v^{\varepsilon}(t)$. From each point $\left(g^{\varepsilon}(t), t\right)$ we can construct a characteristic line $C\left(g^{\varepsilon}(t), t\right)$, which is on the left of the curve $x=g^{\varepsilon}(t) . u \equiv u\left(g^{\varepsilon}(t), t\right)$ on $C\left(g^{\varepsilon}(t), t\right)$. By Lemma $2.2 u$ is monotone with respect to $x$, so $u\left(g^{\varepsilon}(\tau), \tau\right) \leq u\left(g^{\varepsilon}(t), t\right)$ for $\tau<t$, which implies $v^{\varepsilon}$ is increasing monotonically. Let $\varepsilon \rightarrow 0$; then $g^{\varepsilon}(t) \rightarrow g(t), v^{\varepsilon}(t) \rightarrow v(t)$, so $v$ is also increasing monotonically. As a result $v \in B V$, and $\frac{d v}{d t}$ exists as a measure.

We prove by contradiction that $v$ is a continuous function. If $v$ is discontinuous at a point $t_{1}>0$, let $u_{1}=\lim _{t \rightarrow t_{1}+0} v(t), u_{2}=\lim _{t \rightarrow t_{1}-0} v(t)$; then $u_{1}>u_{2}$. Let $t_{2}>t_{1}$; then $v(t) \geq u_{1}$ on $\left(t_{1}, t_{2}\right)$. For each $t \in\left(t_{1}, t_{2}\right)$, if $x>g(t)$ and $x-g(t)$ is small enough, then $u(x, t)>v(t)-\left(u_{1}-u_{2}\right) \geq u_{2}$. We consider the characteristic $C(x, t), \xi=x+f^{\prime}(u(x, t))(\tau-t)$, which doesn't intersect the line segment $\{(x, t) ; t=$ $\left.t_{1}, x>g\left(t_{1}\right)\right\}$, as otherwise we would get $\left.u\right|_{C(x, t)}=u(x, t)>u_{2}$, which contradicts Lemma 2.2. We define $h(t)=\sup \left\{x>g(t) ; x+f^{\prime}(u(x, t))\left(t_{1}-t\right) \leq g\left(t_{1}\right)\right\}$; then $h(t)>g(t)$ for $t>t_{1}$. On the other hand, we consider $x \in(g(t), h(t))$ and the characteristic $C(x, t)$. The slope of $C(x, t)$ is bounded by $f^{\prime}(U)$, so $x-g\left(t_{1}\right) \leq$ $f^{\prime}(U)\left(t-t_{1}\right)$. Besides, $x+f^{\prime}(u(x, t))(\tau-t) \leq h(\tau)$ for $\tau \in\left(t_{1}, t\right)$, because it is also the characteristic $C\left(x+f^{\prime}(u(x, t))(\tau-t), \tau\right)$; then by the definition of $h$, it cannot be greater than $h(\tau)$. Letting $x \rightarrow h(t)$, we get $x+f^{\prime}(u(x-0, t))(\tau-t) \leq h(\tau)$ for $x=h(t)$, which implies $\frac{h(t)-h(\tau)}{t-\tau} \leq f^{\prime}(U)$. Similarly we consider $x>h(t)$ and get 


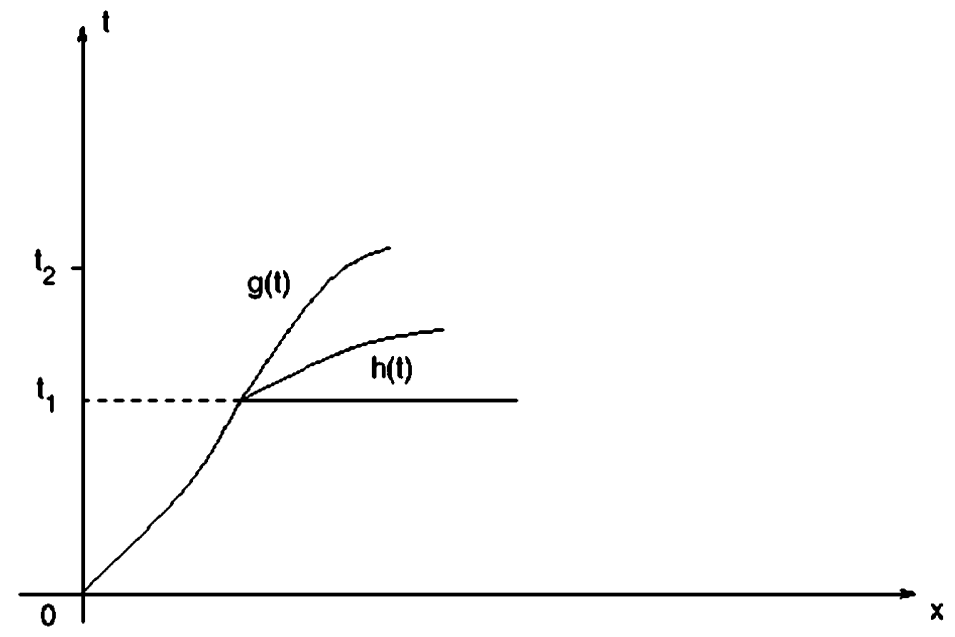

FIGURE 3.

$\frac{h(t)-h(\tau)}{t-\tau} \geq f^{\prime}\left(u_{r}\right)$. Therefore $h(t)$ is continuous and $\lim _{t \rightarrow t_{1}} h(t)=g\left(t_{1}\right)$ (Figure 3). By the definition of $h(t)$, we have $u(h(t)-0, t) \geq u_{1}$ and $u(h(t)+0, t) \leq u_{2}$, so it is a shock. $h^{\prime}(t)$ exists almost everywhere, and by the Rankine-Hugoniot condition

$$
h^{\prime}(t)=\frac{f(u(h(t)-0, t))-f(u(h(t)+0, t))}{u(h(t)-0, t)-u(h(t)+0, t)} .
$$

Given $\varepsilon>0$, let $t>t_{1}$ be small enough; then $u(h(t)-0, t) \leq u_{1}+\varepsilon$. Since we have meas $S=0$,

$$
g(t)=\int_{t_{1}}^{t} g^{\prime}(t) d t+g\left(t_{1}\right)=\int_{t_{1}}^{t} f^{\prime}(v(t)) d t+g\left(t_{1}\right) \geq f^{\prime}\left(u_{1}\right)\left(t-t_{1}\right)+g\left(t_{1}\right) .
$$

Let $\varepsilon$ be small enough; then (18) yields $h^{\prime}(t)<f^{\prime}\left(u_{1}\right)$, and (19) gives $h(t)<g(t)$. This is impossible, so $v$ is continuous. Because $v(t)$ is constant on characteristics and meas $S=0$, we have

$$
v(t)=\int_{0}^{t} v^{\prime}(\tau) d \tau+u_{r}=\int_{S} v^{\prime}(\tau) d \tau+u_{r} \equiv u_{r} .
$$

Because $u$ is a constant on $C\left(x_{0}, t_{0}\right), u\left(x_{0}, t_{0}\right)=u_{r} .\left(x_{0}, t_{0}\right)$ is arbitrary, so $u \equiv u_{r}$ on $\Omega_{+}$.

We consider $\Omega_{-}$next. We define

for $v \geq u_{l *}$.

$$
f^{*}(v)=\frac{f(v)-f\left(u_{r}\right)}{v-u_{r}-q}
$$

Lemma 3.2. If $u \in\left[u_{l_{*}}, v\right]$ on a neighborhood of $\Gamma, v \geq u_{l}^{*}$, then $l^{\prime} \leq f^{*}(v)$.

Proof. We take a sequence $\varepsilon_{1}, \varepsilon_{2}, \cdots, \varepsilon_{k} \rightarrow+0$ as $k \rightarrow \infty$, such that $u\left(l(\cdot)-\varepsilon_{k}, \cdot\right)$ converges weakly. Let $\tilde{u}$ be the limit. Since $u$ is a weak solution on $\left\{(x, t) ; l(t)-\varepsilon_{k}<\right.$ $\left.x<l(t), t_{0}<t<t_{1}\right\}$, where $t_{0}, t_{1}$ are arbitrary, we have

$$
\int_{S}\left\{\left(u_{r}+q\right) l^{\prime}-f\left(u_{r}\right)\right\} d t=\int_{S}\left\{u\left(x-\varepsilon_{k}, t\right) l^{\prime}-f\left(u\left(x-\varepsilon_{k}, t\right)\right)\right\} d t+I_{k},
$$


where $S=\left\{(x, t) ; x=l(t), t_{o}<t<t_{1}\right\}$ and $I_{k}$ is the integral on $t=t_{0}$ and $t=t_{1}$. Since $f^{*}(u) \leq f^{*}(v)$, we have

$$
\begin{aligned}
& \int_{S}\left\{f\left(u\left(x-\varepsilon_{k}, t\right)\right)-f\left(u_{r}\right)-l^{\prime}\left(u\left(x-\varepsilon_{k}, t\right)-u_{r}-q\right)\right\} d t \\
\leq & \int_{S}\left(f^{*}(v)-l^{\prime}\right)\left(u\left(x-\varepsilon_{k}, t\right)-u_{r}-q\right) d t .
\end{aligned}
$$

Let $k \rightarrow \infty$. Then $I_{k} \rightarrow 0$, and we get

$$
\int_{S}\left(f^{*}(v)-l^{\prime}\right)\left(\tilde{u}-u_{r}-q\right) d t \geq 0 .
$$

$\left(\tilde{u}-u_{r}-q\right) d t$ is a measure, denoted by $d \nu$, so

$$
\int_{S}\left(f^{*}(v)-l^{\prime}\right) d \nu \geq 0
$$

Since $S$ is arbitrary, $f^{*}(v)-l^{\prime} \geq 0$ almost everywhere.

We define $\bar{u}$ so that $f^{\prime}(\bar{u})=f^{*}\left(u_{l}^{*}\right)$; then $u_{C J}<\bar{u}<u_{l}^{*}$.

Lemma 3.3. If $u(x, t) \geq \bar{u}$, then $C(x, t)$ intersects $t=0$ at $x<0$, and $g(\tau ; x, t) \leq$ $l(\tau)$ for $\tau \geq 0$. If $u(x, t)<\bar{u}$, then $C(x, t)$ intersects $\Gamma$.

Proof. If $u(x, t) \geq \bar{u}$, let $u^{(1)}=u(x, t)$. If $C(x, t)$ intersects $\Gamma$ at $t_{1} \geq 0$, then

$$
\int_{t_{1}}^{t} l^{\prime} d s>f^{\prime}\left(u^{(1)}\right)\left(t-t_{1}\right) \geq f^{*}\left(u_{l}^{*}\right)\left(t-t_{1}\right) .
$$

There is a point $\left(x_{2}, t_{2}\right)$ in $\left\{(\xi, \tau) ; g(\tau ; x, t)<\xi<l(\tau), t_{1}<\tau<t\right\}$ such that $u\left(x_{2}, t_{2}\right) \geq u_{l}^{*}$. If not, let $v=u_{l}^{*}$ in Lemma 3.2 , then by the conclusion $l^{\prime} \leq f^{*}\left(u_{l}^{*}\right)$, which is impossible. Let $u^{(2)}=u\left(x_{2}, t_{2}\right)$. Since characteristics cannot intersect each other, $C\left(x_{2}, t_{2}\right)$ intersects $\Gamma$, too. We repeat the argument and get $u^{(3)}, \cdots$, such that $f^{*}\left(u^{(k+1)}\right) \geq f^{\prime}\left(u^{(k)}\right) .\left\{u^{(k)}\right\}$ is an increasing sequence, so $\lim _{k \rightarrow \infty} u^{(k)}=u_{\infty}$. If $u_{\infty}<\infty$, then

$$
f^{\prime}\left(u_{\infty}\right) \leq f^{*}\left(u_{\infty}\right)=\frac{f\left(u_{\infty}\right)-f\left(u_{r}\right)}{u_{\infty}-u_{r}-q},
$$

which is impossible. It follows that $u_{\infty}=\infty$, which contradicts the maximum norm estimate, Lemma 2.1 Therefore $C(x, t)$ intersects $t=0$ at $x<0$, and $g(\tau ; x, t) \leq l(\tau)$ for $\tau \geq 0$.

If $u(x, t)<\bar{u}$ and if $C(x, t)$ does not intersect $\Gamma$, then it intersects $t=0$, and $u(x, t)=u_{l}$. This is also a contradiction.

Since $f^{\prime}>0, C\left(x_{0}, t_{0}\right)$ intersects $t=0, x<0$ for all $x_{0}<0, t_{0}>0$, which implies $u\left(x_{0}, t_{0}\right)=u_{l}$. Let $s(t)=\sup \left\{x ; u(x, t)=u_{l}\right\}$ for all $t$; then $s(0)=0$. If $s(t)<l(t)$, then by Lemma $3.3 u(x, t) \leq \bar{u}<u_{l}$ for all $x \in(s(t), l(t)) . x=s(t)$ is a shock wave, because $u$ is discontinuous on it.

Lemma 3.4. $s(t) \equiv l(t)$.

Proof. If not, there is a $t_{1} \geq 0$ such that $s\left(t_{1}\right)<l\left(t_{1}\right)$; then there is a $t_{0}<t_{1}$ such that $s(t)<l(t)$ for $t \in\left(t_{0}, t_{1}\right)$ and $s\left(t_{0}\right)=l\left(t_{0}\right)$. By Lemma $3.2 l^{\prime} \leq f^{*}\left(u_{l}^{*}\right)=f^{\prime}(\bar{u})$, so $l$ is continuous. For $x=s(t)$ we have $u(x-0, t)=u_{l}^{*}$ and $u(x+0, t) \in\left[u_{l_{*}}, \bar{u}\right)$; hence

$$
s^{\prime} \geq \frac{f\left(u_{l}^{*}\right)-f\left(u_{l *}\right)}{u_{l}^{*}-u_{l *}}=f^{\prime}(\bar{u}) .
$$


Consequently

$$
l\left(t_{1}\right)-s\left(t_{1}\right)=\int_{t_{0}}^{t_{1}}\left(l^{\prime}(t)-s^{\prime}(t)\right) d t \leq 0,
$$

which leads to a contradiction.

Obviously we have

Lemma 3.5. $u \equiv u_{l}$ on $\Omega_{-}$.

We are now ready to prove our main result of this section.

Proof of Theorem 1.2, By the Rankine-Hugoniot condition, $l^{\prime}=f^{*}\left(u_{l}\right)$. Therefore the solution is given by (7).

Moreover, by the uniqueness of the limit, the approximate solution $u_{\Delta x}, z_{\Delta x}$ converges in $L^{1}$ to the exact solution $u, z$, given by (7), as $\Delta x \rightarrow 0, \Delta t \rightarrow 0$. The proof is thus completed.

\section{Convergence to weak detonation waves}

We assume that the numerical ignition temperature $U_{i}$ is fixed, the CFL condition (11) holds, and $U_{i}<u_{l *}-q$ to obtain weak detonation waves in this section. In fact the restriction on $U_{i}$ is even stronger. We define a constant $f^{*}$ which satisfies

$$
f^{\prime}\left(u_{l}^{*}\right)>f^{*}>\frac{f\left(u_{l *}\right)-f\left(u_{r}\right)}{u_{l *}-u_{r}-q}=\frac{f\left(u_{l}^{*}\right)-f\left(u_{r}\right)}{u_{l}^{*}-u_{r}-q}=\frac{f\left(u_{l}^{*}\right)-f\left(u_{l *}\right)}{u_{l}^{*}-u_{l *}},
$$

and we show that the constant $u_{w}$ in Theorem 1.3 is $u_{r}+\frac{q f^{\prime}\left(u_{r}\right)}{2 f^{*}}$, which is less than $u_{l *}-q$.

Lemma 4.1. If

$$
u_{r}<U_{i} \leq u_{r}+q f^{\prime}\left(u_{r}\right) \cdot \begin{cases}\frac{\Delta t}{\Delta x}, & \text { for } \frac{\Delta t}{\Delta x} \geq \frac{1}{2 f^{*}}, \\ \frac{1}{f^{*}}-\frac{\Delta t}{\Delta x}, & \text { or } \frac{\Delta t}{\Delta x}<\frac{1}{2 f^{*}},\end{cases}
$$

then

$$
l_{\Delta x}^{\prime}(t) \geq f^{*} .
$$

Proof. By the difference scheme and Lemma 2.4.

$$
\tilde{u}_{j_{0}+1}^{n} \geq u_{j_{0}+1}^{n}+\frac{\Delta t}{\Delta x} f^{\prime}\left(u_{r}\right)\left(u_{j_{0}}^{n}-u_{j_{0}+1}^{n}\right) \geq u_{j_{0}+1}^{n}+\frac{\Delta t}{\Delta x} f^{\prime}\left(u_{r}\right) q .
$$

If $\tilde{u}_{j_{0}+1}^{n}<U_{i}$, then $u_{j_{0}+1}^{n+1}=\tilde{u}_{j_{0}+1}^{n}$. Let $n_{1}$ be a given integer; then we have $z_{j_{0}}^{n_{1}}=0$ and $z_{j_{0}+1}^{n_{1}}=1$. If $z_{j_{0}+1}^{n}$ keeps invariant up to $n_{1}+N$, then

so

$$
\begin{aligned}
U_{i} & >\tilde{u}_{j_{0}+1}^{n_{1}+N-1} \geq u_{j_{0}+1}^{n_{1}}+N \frac{\Delta t}{\Delta x} f^{\prime}\left(u_{r}\right) q \\
& \geq u_{r}+N \frac{\Delta t}{\Delta x} f^{\prime}\left(u_{r}\right) q
\end{aligned}
$$

Let

$$
N<\frac{\left(U_{i}-u_{r}\right) \Delta x}{\Delta t f^{\prime}\left(u_{r}\right) q}
$$

$$
N_{1}=\left[\frac{\left(U_{i}-u_{r}\right) \Delta x}{\Delta t f^{\prime}\left(u_{r}\right) q}\right]+1
$$


then $\tilde{u}_{j_{0}+1}^{n_{1}+N_{1}-1} \geq U_{i}$. If

$$
U_{i} \leq u_{r}+q f^{\prime}\left(u_{r}\right)\left(\frac{1}{f^{*}}-\frac{\Delta t}{\Delta x}\right),
$$

then $N_{1} \leq \frac{\Delta x}{\Delta t f^{*}}$, or $\frac{\Delta x}{N_{1} \Delta t} \geq f^{*}$. (21) follows.

Another possibility is $U_{i} \leq u_{r}+q f^{\prime}\left(u_{r}\right) \frac{\Delta t}{\Delta x}$; then $\tilde{u}_{j_{0}+1}^{n} \geq U_{i}$. If we take $N_{1}=1$, then by the CFL condition (11), $\frac{\Delta x}{\Delta t} \geq f^{*}$. (21) also holds.

Because

$$
\min _{\Delta t / \Delta x}\left(\frac{1}{f^{*}}-\frac{\Delta t}{\Delta x}, \frac{\Delta t}{\Delta x}\right)=\frac{1}{2 f^{*}},
$$

we get (20).

Lemma 4.2. $l^{\prime}(t) \geq f^{*}$.

Proof. Since $l_{\Delta x}^{\prime}$ is bounded, there is a subsequence such that

$$
l_{\Delta x}^{\prime} \rightarrow l^{\prime}\left(L^{p}, p \in(1, \infty)\right)
$$

so $l^{\prime}(t) \geq f^{*}$.

Lemma 4.3. $C(x, t)$ intersects $t=0, x>0$, and $u \equiv u_{r}$ in $\Omega_{+}$.

Proof. Because $u(x, t) \leq U_{i}$ in $\Omega_{+}$, and $f^{\prime}\left(U_{i}\right)<f^{\prime}\left(u_{l *}\right)<f^{*} \leq l^{\prime}(t)$, it follows that $f^{\prime}(u)<l^{\prime}$. $t>0$.

We turn now to consider $\Omega_{-}$. Let $g(t)=\inf \{x ; C(x, t)$ intersects $\Gamma\}$ for all

Lemma 4.4. $g(t)<l(t)$ for all $t>0$, and $g(0)=l(0)=0$.

Proof. If $g\left(t_{0}\right)<l\left(t_{0}\right)$ for one $t_{0}>0$, then $g(t)<l(t)$ for all $t>t_{0}$, because otherwise there is $t_{1}>t_{0}$ be such that $g\left(t_{1}\right)=l\left(t_{1}\right)$ and $g(t)<l(t)$ for $t \in\left[t_{0}, t_{1}\right)$. Let $t_{0}^{\prime}<t_{0}$ such that $g\left(t_{0}^{\prime}\right)=l\left(t_{0}^{\prime}\right)$ and $g(t)<l(t)$ for $t \in\left(t_{0}^{\prime}, t_{0}\right)$. Since $u$ is a weak solution on $\left\{(x, t) ; g(t)<x<l(t), t_{0}^{\prime}<t<t_{1}\right\}$, we have

$$
\begin{aligned}
& \int_{t_{0}^{\prime}}^{t_{1}}\left\{u(g(t)-0, t) g^{\prime}(t)-f(u(g(t)-0, t))\right\} d t \\
= & \int_{t_{0}^{\prime}}^{t_{1}}\left\{(u(l(t)+0, t)+q) l^{\prime}(t)-f(u(l(t)+0, t))\right\} d t .
\end{aligned}
$$

Since $\int_{t_{0}^{\prime}}^{t_{1}} l^{\prime} d t=\int_{t_{0}^{\prime}}^{t_{1}} g^{\prime} d t$, we have

$$
\frac{f\left(u_{l}\right)-f\left(u_{r}\right)}{u_{l}-u_{r}-q}=\frac{1}{t_{1}-t_{0}^{\prime}} \int_{t_{0}^{\prime}}^{t_{1}} l^{\prime}(t) d t .
$$

By Lemma 4.2 the right hand side is greater than or equal to $f^{*}$, which leads to a contradiction.

For any $\varepsilon>0$ there exists $t \in(0, \varepsilon)$ such that $g(t)<l(t)$. Indeed, otherwise $g(t) \equiv l(t)$ for $t \in(0, \varepsilon)$; then $u \equiv u_{l}$ for $x<l(t)$, and by the Rankine-Hugoniot condition $l^{\prime}=f^{*}\left(u_{l}\right)$, which also contradicts Lemma 4.2 . 
Lemmas 4.3 and 4.4 tell us the wave is "supersonic" in front of and behind the discontinuity.

We still don't know if $u(l(t)-0, t)$ exists, so we cannot get a pointwise RankineHugoniot condition on $\Gamma$. However, we can get a weaker statement.

Define $w(t)$ so that

$$
\frac{f(w(t))-f\left(u_{r}\right)}{w(t)-u_{r}-q}=l^{\prime}(t), \quad \text { and } w(t) \in\left(u_{r}+q, u_{l *}\right),
$$

and define $H(u, t)=u l^{\prime}(t)-f(u)$.

Lemma 4.5. If $\varepsilon \rightarrow+0$, then $H(u(l(\cdot)-\varepsilon, \cdot), \cdot)$ converges weak-* to $H(w(\cdot), \cdot)$ in $L^{\infty}$.

Proof. Let $\Omega=\left\{(x, t) ; l(t)-\varepsilon<x<l(t), t_{0}<t<t_{1}\right\}$. Since $u$ is a weak solution on $\Omega$,

$$
\begin{aligned}
& \int_{t_{0}}^{t_{1}}\left\{u(l(t)-\varepsilon, t)-u_{r}-q\right\} l^{\prime}(t) d t \\
= & \int_{t_{0}}^{t_{1}}\left\{f(u(l(t)-\varepsilon, t))-f\left(u_{r}\right)\right\} d t+I_{\varepsilon},
\end{aligned}
$$

where $I_{\varepsilon}$ is the integral on $t=t_{1}$ and $t=t_{0}$. That is,

$$
\int_{t_{0}}^{t_{1}}\{H(u(l(t)-\varepsilon, t), t)-H(w(t), t)\} d t=I_{\varepsilon} .
$$

Let $\varepsilon \rightarrow 0$. Then

$$
\int_{t_{0}}^{t_{1}} H(u(l(t)-\varepsilon, t), t) d t \rightarrow \int_{t_{0}}^{t_{1}} H(w(t), t) d t .
$$

Since $t_{1}, t_{0}$ are arbitrary, convergence follows.

Proof of Theorem [1.3. The condition (20) is weaker then $U_{i} \in\left(u_{r}, u_{w}\right]$, so in fact we have proved the assertions under a slightly weaker condition. The RankineHugoniot condition is in the sense of (22).

We remark that weak detonation waves are not unique, so $\Gamma$ may be a curve, and we don't know if $\lim _{\Delta x \rightarrow 0, \Delta t \rightarrow 0} u_{\Delta x}$ exists. In addition we make more remarks.

Remark 4.1. The condition (20) implies $U_{i}<u_{l *}-q$.

Proof. The condition (20) implies

$$
U_{i} \leq u_{r}+\frac{q f^{\prime}\left(u_{r}\right)}{f^{*}\left(u_{l *}\right)}
$$

Let

$$
\hat{f}=\frac{f\left(u_{l *}\right)-f\left(u_{r}\right)}{u_{l *}-u_{r}}>f^{\prime}\left(u_{r}\right)
$$

then

$$
\begin{gathered}
u_{l *}-q=u_{r}+\frac{q \hat{f}}{f^{*}\left(u_{l *}\right)-\hat{f}}, \\
f^{\prime}\left(u_{r}\right) f^{*}\left(u_{l *}\right)<\hat{f} f^{*}\left(u_{l *}\right)<\hat{f}\left(f^{*}\left(u_{l *}\right)+f^{\prime}\left(u_{r}\right)\right),
\end{gathered}
$$


which gives

$$
\frac{\hat{f}}{f^{*}\left(u_{l *}\right)-\hat{f}}>\frac{f^{\prime}\left(u_{r}\right)}{f^{*}\left(u_{l *}\right)}
$$

Remark 4.2. Let $u_{0}$ satisfy $f^{\prime}\left(u_{0}\right)=f^{*}$. Then $H$ is monotone for $u<u_{0}$.

Proof. We have $\frac{\partial H}{\partial u}(u, t)=l^{\prime}(t)-f^{\prime}(u)$. If $u<u_{0}$, then $f^{\prime}(u)<f^{\prime}\left(u_{0}\right)=f^{*} \leq l^{\prime}(t)$, so $\frac{\partial H}{\partial u}>0$.

From this remark we know that $w \rightarrow H(w, t)$ is one-to-one, but we cannot prove that $u<u_{0}$ near $\Gamma$, so we don't know if $u \rightarrow H(u, t)$ is one-to-one near $\Gamma$. Because of this difficulty, we cannot get the limit of $u$ from Lemma 4.5.

In [12] some sufficient conditions are given to get strong and weak travelling detonation waves of the equation

$$
\frac{\partial(u+q z)}{\partial t}+\frac{\partial f(u)}{\partial x}=\beta \frac{\partial^{2} u}{\partial x^{2}}
$$

and (2). Finally, let us compare the result in [12] and our results. First of all, we recall the results in [12] using our notation.

Given $u_{l}^{*}$ and $u_{l *}$, let

$$
s=\frac{f\left(u_{l}^{*}\right)-f\left(u_{l *}\right)}{u_{l}^{*}-u_{l *}} .
$$

Define $\hat{q}$ by

$$
\frac{f\left(u_{l *}\right)-f\left(U_{i}\right)}{u_{l *}-U_{i}-\hat{q}}=s .
$$

Then there is $q^{C R}$ such that if $q=q^{C R}$, there is a weak detonation travelling wave, and if $q>q^{C R}$, there is a strong detonation travelling wave. $q^{C R}$ can be estimated as follows:

$$
\hat{q}+\frac{K_{0}\left(u_{l *}-U_{i}\right)}{s^{2} C}<q^{C R}<\hat{q}+\frac{K_{0}\left(u_{l *}-U_{i}\right)}{s^{2}}+\frac{2\left(K_{0}\left(u_{l *}-U_{i}\right) \hat{q}\right)^{\frac{1}{2}}}{s},
$$

where $K_{0}=\beta K$.

In our case $\beta \approx f^{\prime} \Delta x, K=\frac{1}{\Delta t}$, so $K_{0}=\beta K \approx f^{\prime} \frac{\Delta x}{\Delta t}$. Let $U_{i}=u_{l *}-q$; then $q=q^{C R}$. By (23)

$$
s\left(q^{C R}-\hat{q}\right)=f\left(u_{l *}\right)-f\left(u_{l *}-q^{C R}\right),
$$

and hence

$$
\begin{aligned}
q^{C R} & =\hat{q}+\frac{1}{s}\left(f\left(u_{l *}\right)-f\left(u_{l *}-q^{C R}\right)\right)=\hat{q}+\frac{f^{\prime} q^{C R}}{s} \\
& =\hat{q}+\frac{f^{\prime}\left(u_{l *}-U_{i}\right)}{s} .
\end{aligned}
$$

By the CFL condition $\frac{\Delta x}{\Delta t}=c_{0} s, c_{0} \geq 1$, so $f^{\prime} \approx K_{o} \frac{\Delta t}{\Delta x}=\frac{K_{0}}{c_{0} s}$. By 25) we get

$$
q^{C R} \approx \hat{q}+\frac{K_{0}\left(u_{l *}-U_{i}\right)}{c_{0} s^{2}} .
$$

This estimate is about the same as the main part of (24). 


\section{ACKNOWLEDGMENTS}

This work was done when the author visited the Department of Mathematics of the University of Wisconsin-Madison as a visiting professor. The author is grate-

ful to the department for their hospitality. He takes special pleasure in thanking Professor S. Jin, who drew his attention to this interesting problem and had some helpful discussions with the author on the manuscript. The author would also like to express his gratitude to the referees for several valuable comments concerning the original manuscript.

\section{REFERENCES}

[1] W. Bao and S. Jin, The random projection method for hyperbolic conservation laws with stiff reaction terms, J. Comput. Phys., 163, 216-248, 2000. MR 2001d:76091

[2] A. C. Berkenbosch, E. F. Kaasschieter and R. Klein, Detonation capturing for stiff combustion chemistry, Combust. Theory Modelling, 2, 313-348, 1998. MR 2000b:80008

[3] R. Courant and K. O. Friedrichs, Supersonic Flow and Shock Waves, Interscience Publishers Inc. New York, 1948. MR 10:637c

[4] M. G. Crandall and A. Majda, Monotone difference approximations for scalar conservation laws, Math. Comp., 34, 1-21, 1980. MR 81b:65079

[5] P. Colella, A. Majda and V. Roytburd, Theoretical and numerical structure for reacting shock waves, SIAM J. Sci. Stat. Comput., 7, 1059-1080, 1986. MR 87i:76037

[6] C. Dafermos, Generalized charateristics and the structure of solutions of hyperbolic conservation laws, Indiana Univ. Math. J., 26, 1097-1119, 1977. MR 56:16151

[7] R. J. DiPerna, Convergence of approximate solutions to conservation laws, Arch. Rat. Mech. Anal., 82, 27-70, 1983. MR 84k:35091

[8] B. Engquist and B. Sjogreen, Robust Difference Approximations of Stiff Inviscid Detonation Waves, CAM Report 91-03 (UCLA 1991).

[9] L. C. Evans, Weak Convergence Methods for Nonlinear Partial Differential Equations, CBMS Regional Conference Series in Mathematics, 74, American Mathematical Society, 1988. MR 91a:35009

[10] D. F. Griffiths, A. M. Stuart and H. C. Yee, Numerical wave propagation in an advection equation with a nonlinear source term, SIAM J. Numer. Anal., 29, 1244-1260, 1992. MR 93h:65111

[11] R. J. LeVeque and H. C. Yee, A study of numerical methods for hyperbolic conservation laws with stiff source terms, J. Comput. Phys., 86, 187-210, 1990. MR 90k:76009

[12] A. Majda, A qualitative model for dynamic combustion, SIAM J. Appl. Math., 41, 70-93, 1981. MR 82j:35096

[13] R. B. Pember, Numerical methods for hyperbolic conservation laws with stiff relaxation, I: Spurious solutions, SIAM J. Appl. Math., 53, 1293-1330, 1993. MR 95a:65173

[14] L.-a. Ying and Z.-h. Teng, A hyperbolic model of combustion, North Holland Mathematics Studies, 81, North-Holland, Amsterdam, and Kinokuniya, Tokyo, 409-434, 1983. MR 86a:35092

School of Mathematical Sciences, Peking University, People's Republic of China 